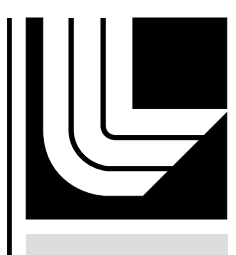

LAW RENCE LIVERMORE N A TIO N A L LABORATORY

\title{
UCRL-TR-208848
}

\section{A Comparison of Digital Signal Extraction Techniques}

M. Cunningham, F. Dowla

January 5, 2005 
This document was prepared as an account of work sponsored by an agency of the United States Government. Neither the United States Government nor the University of California nor any of their employees, makes any warranty, express or implied, or assumes any legal liability or responsibility for the accuracy, completeness, or usefulness of any information, apparatus, product, or process disclosed, or represents that its use would not infringe privately owned rights. Reference herein to any specific commercial product, process, or service by trade name, trademark, manufacturer, or otherwise, does not necessarily constitute or imply its endorsement, recommendation, or favoring by the United States Government or the University of California. The views and opinions of authors expressed herein do not necessarily state or reflect those of the United States Government or the University of California, and shall not be used for advertising or product endorsement purposes.

This work was performed under the auspices of the U.S. Department of Energy by University of California, Lawrence Livermore National Laboratory under Contract W-7405-Eng-48. 


\title{
A Comparison of Digital Signal Extraction Techniques
}

\author{
Mark Cunningham and Farid Dowla \\ Lawrence Livermore National Laboratory \\ P. O. Box 808, Livermore, CA 94551-0808
}

\begin{abstract}
We compare the performance of two methods of digital filtering to detect a radioactive source moving past a gamma-ray sensor. The first method is the box-car filter, which is a standard method used in the detection of a moving radioactive source. The second method is the matched filter, which takes into account the variation in the number of photons absorbed in a gamma-ray sensor as a source moves past the sensor. We optimize both methods to detect a source moving at 5, 10, 15 and $20 \mathrm{mph}$, and the receiver-operator characteristics of the two techniques are plotted for comparison. The improvement of the matched filter over the box car filter is $27 \%$ at $5 \mathrm{mph}$ and $22 \%$ at 10 mph for a $90 \%$ probability of detection and an average hours between false alarms equal to 10 .
\end{abstract}

\section{Introduction}

The process of detecting of a radioactive source moving past a radiation sensor is a problem that is well suited for the use of digital signal extraction techniques. Examples of sources we are interested in detecting include the movement of a radiological dispersal device and the illicit movement of industrial radioactive sources. The process of detecting a moving radioactive source is composed of four parts: measurement, detection, classification and estimation. The measurement component of the radiation detection process is accomplished with the use of a radiation sensor. The sensor, which includes an absorbing material and electronics, measures the properties of the gamma-radiation, i.e. the energy of the radiation, time of arrival or whatever property the sensor measures. The second step in the radiation detection process is the analysis of the measured radiation properties to make a decision that a radioactive source did or did not move past the radiation sensor. This second step is called detection. The third step is classification, which is the determination of the taxonomy of the source. The taxonomy can be broken down into the type of radiation, the isotope, the shielding, etc. The final step in the radiation detection process is called estimation. In this step, an algorithm uses the information from the classification step to estimate the properties of the material present, including the mass or age of the material. In this paper we focus our attention on the detection part of the radiation detection process.

Several digital signal extraction algorithms have been used for the extraction of signal from a gamma-ray sensor output of a radiation detection system using a timebinned method of data acquisition. In this standard method of data acquisition, the aggregate properties of photons are measured since the acquisition interval is long compared to the inter-arrival time of photons. Algorithms have been written that do a comparison of the most recently acquired data to the data acquired previously. An example of this type of algorithm is the Single Interval Test (SIT). Other algorithms use filtering to reduce the variance of the signal before the comparison between the most recently acquired data and the previously acquired data is performed. Examples of these 
filtering techniques include: an exponential smoothing filter, ${ }^{1}$ a moving average filter, ${ }^{2}$ an S-fold scaler, ${ }^{3}$ a stepwise monitor, ${ }^{4}$ and the sequential probability ratio test (SPRT). ${ }^{4}$

In a paper by Fehlau ${ }^{5}$, the performance of the exponential smoothing filter, the moving average filter and SPRT was measured. The performance of these filters was shown to be equal for the case that each filter was optimized for the particular detection problem. In the Fehlau paper, a source was moved past the radiation detection system at a know velocity; therefore, each algorithm could be optimized for the particular detection problem. A more generic problem would be the detection of an unknown source at an unknown velocity. The addition of a velocity sensor would then allow the adaptive filtering of data so that the detection system would be optimized for a source moving past the detection system at any velocity. To implement the velocity information in the detection algorithm would require both an adaptive method for time-binning data and an adaptive algorithm for the detection of the radioactive source.

For the filters used previously for the detection of a moving radioactive source, a limited amount of information about the signal was used for the optimization of the detection algorithm. For example, the approximate time the source was close enough to the detection system for the radiation from the source to be absorbed in the sensor was used to set the width of the moving-average filter or box-car filter. Also, the acquisition time width was varied to maximize the probability of detection; however, this optimization method made the assumption that the source strength was: zero before the source moved near the sensor; constant while the source was in front of the sensor; and zero after the source moved away from the sensor. A more realistic approach would assume that the signal increases as the source moves towards the radiation sensor and then decreases as the source moves away from the sensor. In this paper, we examine the benefit of using the variation in the number of photons absorbed in the gamma-ray sensor as a source moves past the sensor, which is called a physics-based model or a matched filter. We determine the benefit of the use of a matched filter by doing a comparison between the matched filter and the box-car.

\section{Review of Statistical Decision Methods}

The problem of detecting a radioactive source moving past a radiation sensor is a general problem in radiation detection. This detection problem is similar to the detection of a weak signal in noise, where the signal is the radiation captured from the source and the noise is the background radiation. Here we are being careful to refer to the hardware (absorbing material and electronics) as a sensor. We reserve the term "detector" for the algorithm used to determine if a source has moved past the radiation sensor. This detection problem fits well into the simple hypothesis-testing category of statistical decision theory, where the probability density functions (PDFs) of both the signal (source) and noise (background) are well known.

There are primarily two types of detectors that can be used for this type of detection problem: the Neyman-Pearson (NP) and the Bayesian methods. ${ }^{6-7}$ For the NP method, no prior knowledge is used to determine weather a detection has occurred, whereas in the Bayesian method, prior knowledge of the probability of occurrence of the various hypotheses is used. For the Bayesian method, we can minimize the probability of making an incorrect decision, where an incorrect decision is either deciding a source was present when there was no source, or deciding a source was not present when there was a 
source present. In the detection of a source moving past a sensor, we do not know beforehand what the probability of a source being present is; therefore, in our detection problem we choose the NP method for our detector.

The NP detector is the detector that maximizes the probability of detection for a given probability of false alarm. To use this method, it is required that the PDFs of both the signal and noise are known. For our detection system, there are two hypotheses: hypothesis 1 , or $H_{1}$, that no source is present; and hypothesis 2 , or $H_{2}$, that a source is present. For the NP test, the ratio of the probabilities of these two hypotheses is compared to a threshold. Each hypothesis is dependent on a set of data; therefore, we write: $L(\vec{x})=p\left(\vec{x} ; H_{2}\right) / p\left(\vec{x} ; H_{1}\right)>\gamma$, where $\vec{x}$ is the vector that represents the data that is used to test each hypothesis, $p\left(\vec{x}, H_{i}\right)$ is the probability that $H_{i}$ is true give the data $\vec{x}$, and $\gamma$ is the threshold used to decide if $H_{1}$ or $H_{2}$ is more likely. The test, $L(\vec{x})$, is call the likelihood ratio test and it is compared to the threshold $\gamma$, where $\gamma$ is found by:

$$
P_{F A}=\int_{\{\bar{x}: L(x \bar{x})>\gamma\}} p\left(\vec{x} ; H_{1}\right) d \vec{x},
$$

where $\{\vec{x}: L(\vec{x})>\gamma\}$ is the set of values of $\vec{x}$ for which $L(\vec{x})>\gamma$, and $P_{F A}$ is the false alarm probability that is determined to be acceptable to the detection problem. Once the threshold is determined using (1), then the probability of detection can be found using:

$$
P_{D}=\int_{\{\bar{x}: L(\vec{x})>\gamma\}} p\left(\vec{x} ; H_{2}\right) d \vec{x} \text {. }
$$

As an example of a detection problem, we take the simple case of measuring the number of counts from a radiation sensor for a period of time $\Delta t$. We want to decide if the measured number of counts is more likely to come from source 2, with expected number of counts $A_{2}$, or from source 1 , with expected number of counts $A_{1}$. The NP likelihood ratio is

$$
L(\vec{x})=\frac{p\left(\vec{x} ; H_{2}\right)}{p\left(\vec{x} ; H_{1}\right)}=\frac{A_{2}^{x} e^{-A_{2}} / x !}{A_{1}^{x} e^{-A_{1}} / x !}=\left(A_{2} / A_{1}\right)^{x} e^{\left(-A_{2}+A_{1}\right)}>\gamma .
$$

Since $A_{1}$ and $A_{2}$ are known constants, we can move them to the right side of the equation using the natural log function, then we find $x>\left(\ln \gamma+\left(A_{2}-A_{1}\right)\right) / \ln \left(A_{2} / A_{1}\right)=\gamma^{\prime}$. The value on the left is a function of the measured data and is called the test function, $T(\vec{x})=x$. Note that the left side of the equation contains no unknown parameters. The right side of the equation is the modified threshold, which is found by

$$
P_{F A}=\int_{\left\{\bar{x}: T(\bar{x})>\gamma^{\prime}\right\}} p\left(\vec{x} ; H_{1}\right) d \vec{x}=\sum_{x>\gamma^{\prime}}^{\infty} \frac{A_{1}^{x} e^{-A_{1}}}{x !},
$$

and the probability of detection is

$$
P_{D}=\int_{\left\{\vec{x}: T(\vec{x})>\gamma^{\prime}\right\}} p\left(\vec{x} ; H_{2}\right) d \vec{x}=\sum_{x>\gamma^{\prime}}^{\infty} \frac{A_{2}^{x} e^{-A_{2}}}{x !} .
$$

For this example, we compare each measurement of the number of counts for a period of time $\Delta t$ to $\gamma^{\prime}$. If the number of counts is greater than $\gamma^{\prime}$, then hypothesis 2 , source 2 , is declared. 
For many detection situations, we are able to make many measurements of the same parameter in order to improve the detection of a signal. As an example, let's take the simple case of measuring the number of counts from a radiation sensor for a period of time $\Delta t$, then we repeat the measurement so that we take the data $N$ times. We want to decide if the measured number of counts for the series of measurements is more likely to come from source 2, with expected number of counts $A_{2}$, or from source 1 , with expected number of counts $A_{1}$. The NP likelihood ratio test for this example is

$$
L(\vec{x})=\frac{p\left(\vec{x} ; H_{2}\right)}{p\left(\vec{x} ; H_{1}\right)}=\left(\prod_{n=0}^{N-1} A_{2}^{x_{n}} e^{-A_{2}} / x_{n} !\right) /\left(\prod_{n=0}^{N-1} A_{1}^{x_{n}} e^{-A_{1}} / x_{n} !\right)>\gamma .
$$

After some simplification, (5) becomes

$$
T(\vec{x})=<\vec{x}>=1 / N \sum_{n=0}^{N-1} x_{n}>\left(\ln \gamma+N\left(A_{1}-A_{2}\right)\right) /\left(N \ln \left(A_{2} / A_{1}\right)\right)=\gamma .
$$

The test function for this case, $T(\vec{x})=\langle x\rangle$, is the average value of the data. This is the optimal test statistic for this case. The right side of the equation is the modified threshold, which is found by

$$
P_{F A}=\int_{\left\{\bar{x}: T(\vec{x})>\gamma^{*}\right\}} p\left(\vec{x} ; H_{1}\right) d \vec{x}=\sum_{x_{0}^{*}}^{\infty} \sum_{x_{1}^{*}}^{\infty} \cdots \sum_{x_{N-1}^{*}}^{\infty} \prod_{n=0}^{N-1} \frac{A_{1}^{x_{n}} e^{-A_{1}}}{x_{n} !},
$$

where $\vec{x}^{*}$ is the set of beginning points for the summations in (7), and the probability of detection is

$$
P_{D}=\int_{\left\{\bar{x}: T(\bar{x})>\gamma^{\prime}\right\}} p\left(\vec{x} ; H_{2}\right) d \vec{x}=\sum_{x_{0}^{*}}^{\infty} \sum_{x_{1}^{*}}^{\infty} \ldots \sum_{x_{N-1}^{*}}^{\infty} \prod_{n=0}^{N-1} \frac{A_{2}^{x_{n}} e^{-A_{2}}}{x_{n} !} .
$$

For this example, we compare the average number of counts in time $\Delta t$ to $\gamma^{`}$. If the average number of counts is greater than $\gamma^{\prime}$, then hypothesis 2 , source 2 , is declared.

\section{Optimal Test Function}

Now we want to derive the case of a deterministic signal, which corresponds to a source moving past the radiation sensor. For the deterministic signal, we know all the parameters of the signal, i.e. velocity, time of pass by, strength of source, etc. For hypothesis 1, that no source is present, the expected number of counts per time $\Delta t$ is $A_{1}$. For hypothesis 2, we expect that the number of counts per time bin will be $A_{1}+s[n]$, where $s[n]$ is the contribution of the source at data point n. The NP likelihood ratio is

$$
L(\vec{x})=\frac{p\left(\vec{x} ; H_{2}\right)}{p\left(\vec{x} ; H_{1}\right)}=\left(\prod_{n=0}^{N-1}\left(A_{1}+s[n]\right)^{x_{n}} e^{-\left(A_{1}+s[n]\right)} / x_{n} !\right) /\left(\prod_{n=0}^{N-1} A_{1}^{x_{n}} e^{-A_{1}} / x_{n} !\right)>\gamma .
$$

For the case that $s[n]=A_{2}-A_{1}$ for all $n$, (9) reduces to (5). After some algebra, we can write the test statistic for this case as

$$
T(\vec{x})=\sum_{n=0}^{N-1} x_{n} \ln \left(1+\frac{s[n]}{A_{1}}\right)>\gamma^{`} .
$$

Each of the measured data points, $x_{n}$, is multiplied by a weighting function, $\ln \left(1+s[n] / A_{1}\right)$, that depends on the expected strength of the source and the background. All parameters of $s[n]$ must be known in order to use (10) as a test statistic, . If there are 
unknown parameters, than a different approach must be used for the detection problem. An example of a possible method is the Generalized Likelihood Ratio Test. ${ }^{6-7}$ The test statistic given as equation (10), is the optimal test statistic for the signal $s[n]$ and the Poisson PDF of both the signal and the noise.

\section{Modeling the Radiation Source for Signal Processing}

We want to be able to determine the values of $s[n]$ that will be put into the test statistic (10). For the case of a point source moving at velocity, $v$, past a radiation sensor at a distance $D$ from the face of the sensor, we expect that the number of counts in the sensor for a time $\Delta t$ is

$$
s^{`}[n]=A_{1}+s[n]=A_{1}+A_{3} \int_{n \Delta t}^{(n+1) \Delta t}\left[\int \frac{D^{2}}{\left(D^{2}+y^{2}+\left(x-\left(x_{0}+v t\right)\right)^{2}\right)^{3 / 2}} d \sigma\right] d t,
$$

where $A_{1}$ is the number of counts due to background, $d \sigma$ is the integration over the surface of the sensor, $A_{3}$ is the expected number of counts in $4 \pi$ during time $\Delta t, x_{0}$ is the initial position of the source, and $n=0,1 \ldots, N-1$. We have assumed that the source moves parallel to the face of the sensor and only in the $\hat{x}$ direction. Also, we have assumed that only the front face of the sensor collects photons, and we assume that the solid angle is the only variable in the reduction of the number of photons entering the sensor. Hence, we ignore shielding near the detector and the loss of photons due to air attenuation.

We now need to determine the value of $x_{0}$. There are two extremes for the case of a source passing by a detector. The first is that the source is directly in front of the detector at the midpoint of the integration for $s[n]$. This will maximize the number of counts possible in any time bin. The second case is if the source is directly in front of the detector at either end of the integration for $s[n]$. This second case will result in the peak rate from the source being divided between two time bins and is a worst case since the maximum number of counts in any bin will be less than in the first case. In reality, the situation will be evenly distributed between these two cases.

In order to implement the worst case, we need the total number of data points, $N$, to be even. For the best case, the total number of data points will be odd. Independent of the two methods, the initial position of the source is $x_{0}=-v N \Delta t / 2$. Therefore, we have

$$
s[n]=A_{3} \int_{n \Delta t}^{(n+1) \Delta t}\left[\int \frac{D^{2}}{\left(D^{2}+y^{2}+(x-v(t-N \Delta t / 2))^{2}\right)^{3 / 2}} d \sigma\right] d t .
$$

For the case that the dimensions of the detector are small compared to the closest pass by distance of the source, we can simplify this to

$$
s[n]=A_{3} \int_{n \Delta t}^{(n+1) \Delta t} \frac{\Sigma}{4 \pi\left(D^{2}+v^{2}(t-N \Delta t / 2)^{2}\right)} d t,
$$

where $\Sigma$ is the area of the detector. It is more convenient to state the expected number of photons that will be absorbed in the detector than to state the expected number of photons that are emitted into $4 \pi$; therefore, we write $A_{2}=A_{3} \Sigma \Delta t / 4 \pi D^{2}$, and 


$$
s[n]=\frac{A_{2} D^{2}}{\Delta t} \int_{n \Delta t}^{(n+1) \Delta t} \frac{1}{\left(D^{2}+v^{2}(t-N \Delta t / 2)^{2}\right)} d t,
$$

where $A_{2}$ is the expected number of photons from the source that are absorbed in the detector in the time $\Delta t$ when the source is stationary and directly in front of the sensor.

\section{Box-Car Filter}

The box-car filter is an averaging technique that reduces the variance in the number of counts per time bin by weighting each point in the average equally. To apply the box-car filter to a data set of $N$ points, each point in the data set is averaged with the $N-1$ nearest neighbors, where $N$ is an odd number greater than or equal to 3 . Then $x_{i}=\left(\sum_{n=i-(N-1) / 2}^{i+(N-1) / 2} x_{n}\right) / N$, where $x_{i}^{\prime}$ is the filtered data and $x_{n}$ is the unfiltered data. This weighting of the data is the test statistic shown in (6).

\section{Matched Filter}

The matched filter is similar to the box-car filter in that each data point is averaged with a set of nearest neighbors; however, each data point is multiplied by the expected weight of each time bin. Then $x_{i}=\left(\sum_{n=i-(N-1) / 2}^{i+(N-1) / 2} a_{n} x_{n}\right) / N$. In the box-car filter $a_{n}=1$ for all $n$. The weighting of the data for the matched filter is $a_{n}=\ln \left(1+s[n] / A_{1}\right)$, where $s[n]$ is given by (14).

\section{Simulation}

We are now ready to calculate the difference between the box-car filter and the matched filter. For the simulation of a source moving past a radiation sensor, we assume a background count rate of 100 photons/second and a source count rate of 35 counts/second. The source moves past the sensor at $10 \mathrm{mph}$, and the closest approach of the source to the face of the sensor is 10 feet. The data acquisition rate is $\Delta t=0.6$ seconds, and the total number of bins in each filter $=3$. With these parameters, we calculate the probability of false alarm and probability of detection for both filters. These values are plotted in Figure 1.

We can state that the matched filter performs better than the box-car filter since the probability of detection of the source is higher for the matched filter than the box-car filter for all values of the probability of false alarm; however, for this simulation, neither filter has been optimized for the detection situation proposed. In order to optimize each filter for this detection problem, a Monte Carlo simulation was used to vary the number of bins and the acquisition time per bin for each filter to maximize the probability of detection. A Monte Carlo simulation was used instead of calculating the probability of detection and probability of false alarm as described in the statistical decision theory section due to the long time required to calculate each algorithm. For the detection problem in the previous paragraph, the optimized parameters for both filters are shown in Table 1. In order to optimize each filter, a simulated source moved past the sensor, and many parallel filters were used to detect the source. For both the box-car filter and the 
matched filter, the acquisition time, $\Delta \mathrm{t}$, was allowed to vary from 0.05 to 10 seconds, and the filter width was allowed to vary from $3 \Delta$ t to 10 seconds.

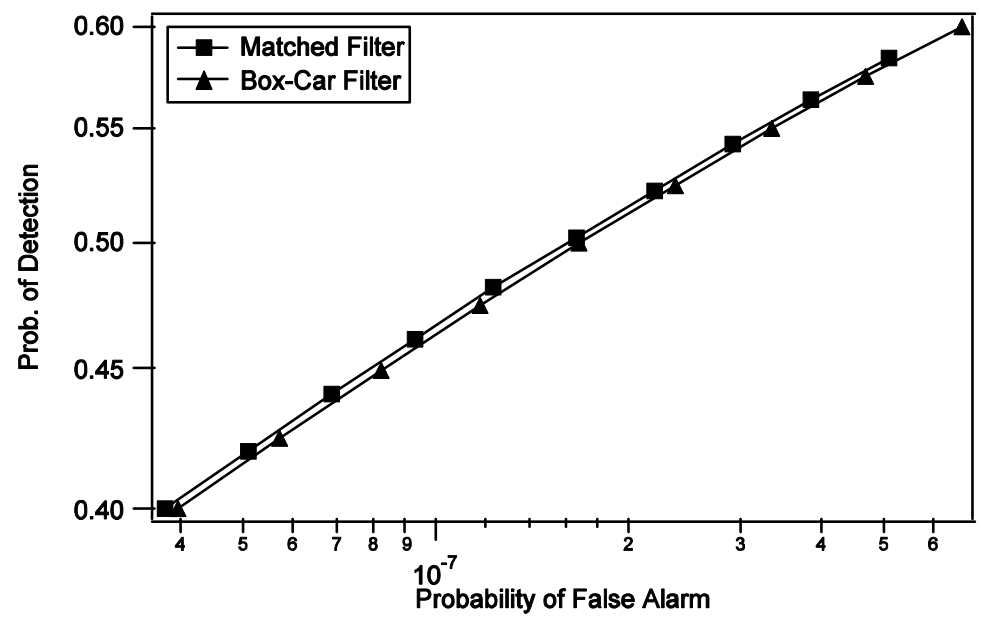

Figure 1: Probability of Detection versus Probability of False Alarm for both the Box-Car Filter and Matched Filter.

Table 1: Optimized parameters for the Box-Car and Matched Filters for $10 \mathrm{mph}$.

\begin{tabular}{|c|c|c|}
\hline Filter & $\Delta \mathrm{t}$ (seconds) & Filter Width (seconds) \\
\hline Box- Car & 0.6 & 1.8 \\
\hline Matched & 0.05 & 1.65 \\
\hline
\end{tabular}

The optimized filter width of both the box-car and matched filters is approximately the same, as shown in Table 1 . This similarity in the filter width is due to the same length of time that the radiation sensor absorbs photons from the source for each filter. A longer filter window would decrease the signal (source) to noise (background) ratio by increasing the amount of noise measured. A shorter filter window would decrease the signal to noise ratio by decreasing the amount of signal measured. There is, however, a difference in the data acquisition time for the two filters. For the box-car filter, there is no added information to be used if the data acquisition rate is increased from the minimum number of data bins. After the acquisition of the data, all the data bins are averaged together and any information that is inherent in the data from a faster data acquisition system is lost. For the matched filter, however, the information that is inherent in the data from a faster data acquisition system is used since the matched filter depends on the signal shape.

It should be noted that for the matched filter, the number of data bins is 33. This large number of data bins creates a problem with doing calculations with the procedure from the statistical decision theory section due the constraints on processing power of a computer. There are several other problems with the statistical decision theory procedure for calculating the probability of false alarm and detection, including: it does not take into account the variation in the calculation of the background due to the finite length of time the background is measured in a real radiation sensor; and it does not take into account the variation in the relative start of a time bin to the time of the peak radiation count rate during a pass by of the source, which should be random and uniform. Due to these three problems, we choose to do calculations of the probabilities of detection and false alarm 
using a Monte Carlo method; however, it should be noted that the section on statistical decision theory is still useful since it allowed us to determine the optimal test statistic for the detection problem of a radioactive source moving past a radiation sensor. The optimal test statistic is the matched filter.

A Monte Carlo simulation was used instead of calculating the probability of detection and probability of false alarm using the statistical decision theory. A source was simulated moving past the sensor at 5, 10, 15 and $20 \mathrm{mph}$, and for a closest approach of 10 feet. The background count rate was 500 counts/second and the source count rate was 95 (5mph), 135 (10mph), $155(15 \mathrm{mph})$ and $180(20 \mathrm{mph})$ counts/second. For both the box-car filter and the matched filter, the acquisition time was varied from 0.05 to 10 seconds in steps of 0.05 seconds, and the filter width was allowed to vary from $3 \Delta$ t to 10 seconds in steps of $2 \Delta \mathrm{t}$. Only the acquisition time and filter width that maximized the probability of detection was used in the final simulation. The background acquisition time was 60 seconds, and the start position of the source was varied so that source was directly in front of the detector between the midpoint of the integration for $s[n]$ and the end of the integration for $s[n]$.

The information in Figure 1 is not the ideal way to compare the performance of each detection algorithm due to the difference in the data acquisition time for each detection algorithm. Also, we are not only interested in stating the probability of false alarm, we are also interested in the absolute comparison of the detection algorithms. Instead of plotting the probability of detection versus the false alarm probability, we choose to plot the probability of detection versus the average hours between false alarms, as shown in Figure 2 for the box-car filter and a source moving past the sensor at 10 and $20 \mathrm{mph}$. Also shown in Figure 2 are error bars for the probability of detection and error bars for the average hours between false alarms. The errors bars for the probability of detection and the error bars for small values of the average hours between false alarms are too small to be seen on this graph. The error bars for the probability of detection depend on the number of times the source is simulated moving past the sensor, and the error bars for the average hours between false alarms depends on the total length of time the simulations are run.

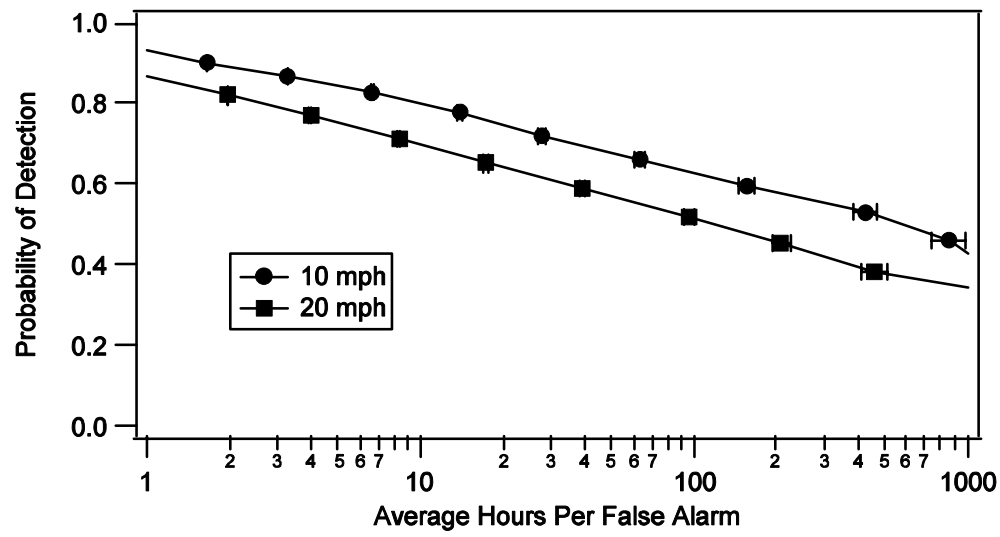

Figure 2: Probability of Detection versus Average Hours Between False Alarms for the Box-Car Filter at 10 and $20 \mathrm{mph}$. 
For the data shown in Figure 2, the probability of detection decreases as the average hours between false alarms increases. This decrease in detection probability is due to the decrease in the sensitivity of the detection algorithm as the detection algorithm is made less prone to false alarms. Also of interest for this simulation is the absolute source strength that the detection algorithm will detect for the scenario described above. Shown in Figure 3 is the probability of detection versus source count rate for a source moving past a radiation detection system at 10 and $20 \mathrm{mph}$ and using a box-car filter. The value of the source count rate is the number of photons per second that would be absorbed in the sensor if a radiation source was directly in front of the sensor and not moving. In this simulation, a value of 10 hours between false alarms was used. For small values of source count rate, the box-car filter does not detect the source at either velocity. At high values of source count rate, the box-car filter is able to detect the source at both velocities. The transition from a low probability of detection to a high probability of detection occurs at a smaller value of source count rate for the lower velocity due to the longer time the source spends in front of the detection system.

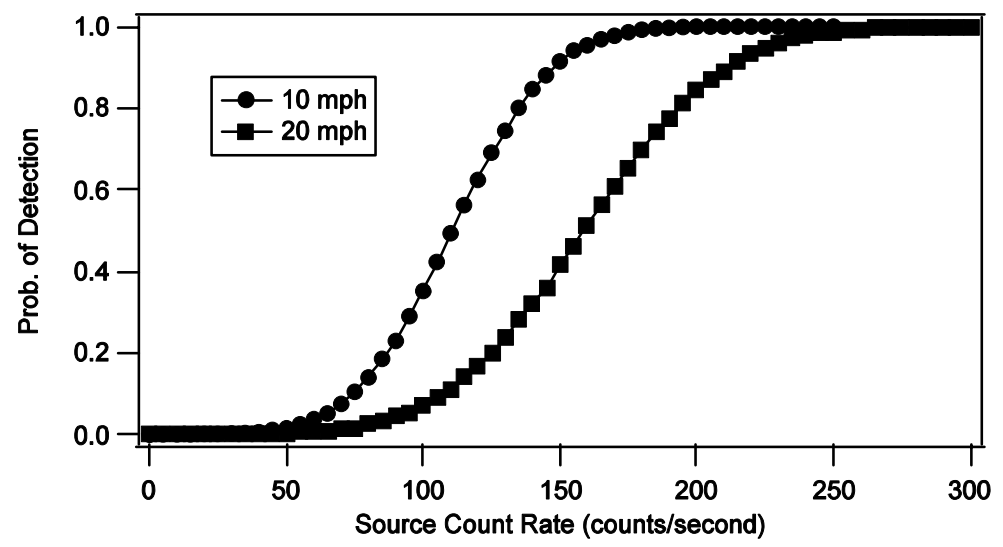

Figure 3: Probability of Detection versus Source Count Rate for the Box-Car Filter at 10 and 20 mph.

We now do a direct comparison between the box-car filter and the matched filter. Shown in Figures 4 and 5 are the probability of detection for both filters as a function of the average hours between false alarms at 5 and $10 \mathrm{mph}$. The matched filter performs better than the box-car filter since the probability of detection for the matched filter is higher for the matched filter than the box-car filter for all average hours between false alarms. The data for the matched filter does not extend out to large values of the average hours between false alarms due to the longer processing time of the matched filter. This also accounts for the larger error bars on the matched filter than the box-car filter for the average hours between false alarms.

A further comparison between the matched filter and box-car filter is shown in Figure 6. This comparison of the probability of detection versus source count rate for both filters is processed for an average hours between false alarms of 10. From Figure 4 and 5, it is evident that the performance difference between the two filters would be larger at a larger value of the average hours between false alarms; however, the error bars for the average hours between false alarms also becomes larger. The improvement of the matched filter over the box car filter, shown in Table 2, is $27 \%(22 \%)$ at $5(10) \mathrm{mph}$ for a $90 \%$ probability of detection, and the shape of the curve is different for the matched filter than the box-car filter. The matched filter has a steeper transition from a low probability 
of detection to a high probability of detection, which is indicative of a better detection algorithm.

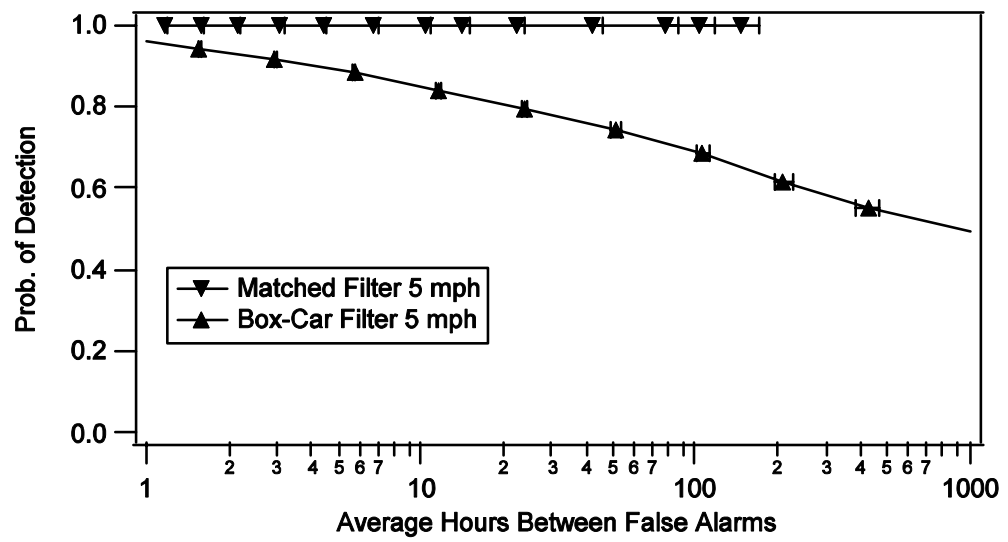

Figure 4: Probability of Detection versus Average Hours Between False Alarm for both the Box-Car and Matched Filters at $5 \mathrm{mph}$.

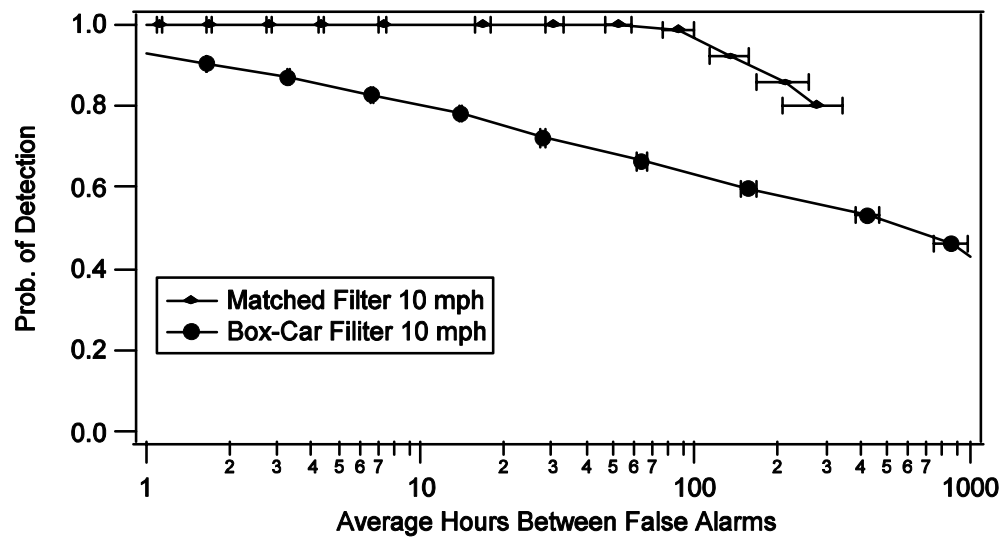

Figure 5: Probability of Detection versus Average Hours Between False Alarms for both the Box-Car and Matched Filters at $10 \mathrm{mph}$.

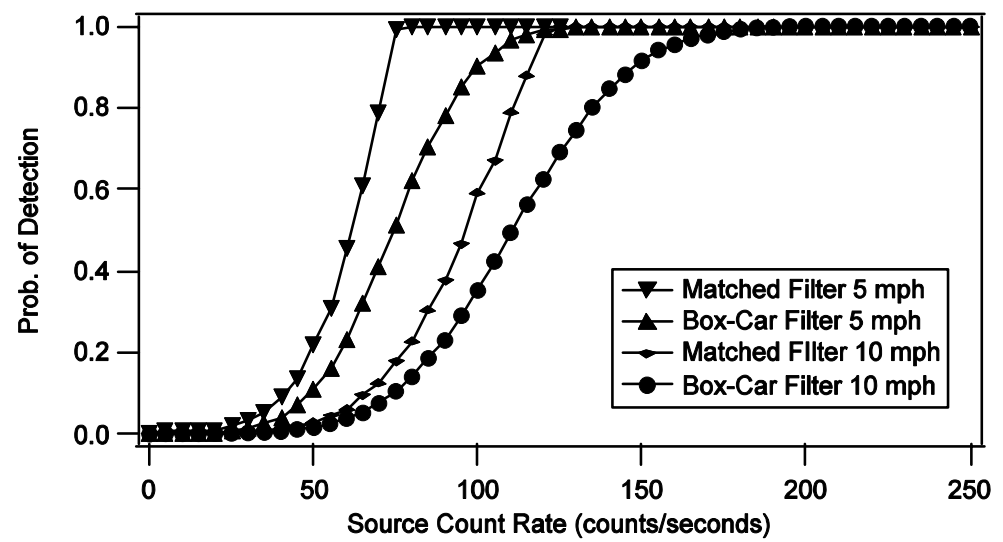

Figure 6: Probability of Detection versus Source Count Rate for both the Box-Car and Matched Filters at 5 and $10 \mathrm{mph}$. 
Table 2: Performance difference between the Box-Car filter and matched filter.

\begin{tabular}{|l|c|c|}
\hline Filter & $\begin{array}{c}\text { Source Rate for 90\% Prob. } \\
\text { of Detection at } 5 \mathrm{mph}\end{array}$ & $\begin{array}{c}\text { Source Rate for 90\% Prob. } \\
\text { of Detection at } 10 \mathrm{mph}\end{array}$ \\
\hline Box-Car & 100 & 148 \\
\hline Matched & 73 & 116 \\
\hline
\end{tabular}

\section{Conclusion}

We compared the use of a matched filter to a box-car filter to detect a radioactive source moving past a radiation detection system. The matched filter takes into account the signal shape as the radioactive source moves past the radiation detection system. The improvement of the matched filter over the box car filter was $27 \%$ at $5 \mathrm{mph}$ and $22 \%$ at $10 \mathrm{mph}$ for a $90 \%$ probability of detection and an average hours between false alarms equal to 10 .

\section{References}

1. P. Dumesnil and J. L. Greco, "Optimization of the processing of the counts from a doorway monitor for radioactive substances," Proc. $7^{\text {th }}$ Ann. ESARDA Symp. Safeguards Nuc. Mater. Manag., Liege, Belgium, May 21-23, 1985.

2. W.H. Chambers, C. N. Henry, H. F. Atwater, W. E. Kunz, P. E. Fehlau, T. E. Sampson, R. D. Hastings, T. H. Whittelsey, and G. M. Worth, "Portal Monitor for Diversion Safeguards," Los Alamos Scientific Laboratory report LA-5681 (1974).

3. E. J. Dowdy, C. N. Henry, R. D. Hastings, and S. W. France, "Neutron Detector Suitcase for the Nuclear Emergency Search Team," Los Alamos Scientific Laboratory report LA-7108 (1978).

4. P. E. Fehlau, J. C. Pratt, J. T. Markin and T. Scurry, Jr., "Smarter radiation monitors for safeguards and security,” Nucl. Mat. Man., vol. XII, p. 122, 1983.

5. P. E. Fehlau, "Comparing A Recursive Digital Filter with the Moving-Average and Sequential Probability-Ratio Detection Methods for SNM Portal Monitors," IEEE Tans. On Nucl. Sci., vol. 40, no. 2, p. 143-146, April 1993.

6. Steven Kay, "Fundamentals of Statistical Signal Processing,” Vol. II, Prentice Hall PTR, Upper Saddle River, New Jersey, 1998.

7. Harry L. Van Trees, "Detection, Estimation, and Modulation Theory," Part I, John Wiley \& Sons, Inc., New York, New York, 2001. 